ARTÍCULO ORIGINAL

\title{
Caracterización de la curva de lactancia y rendimiento en cabras Saanen de un tambo semi-intensivo de la provincia de Buenos Aires, Argentina
}

\author{
Steffen $\mathrm{KD}^{1,3^{*}}$, Arias $\mathrm{RO}^{3}$, Gortari $\mathrm{L}^{2}$, Moré $\mathrm{G}^{1,2}$ \\ ${ }^{1}$ Laboratorio de Inmunoparasitología, Facultad de Ciencias Veterinarias, Universidad Nacional de La \\ Plata, Argentina. \\ ${ }^{2}$ Comisión Nacional de Investigaciones Científicas y Técnicas (CONICET), Argentina. \\ 3 Cátedra de Introducción a la producción animal, FCAyF-UNLP, La Plata (1900), Buenos Aires, \\ Argentina, Argentina.
}

* Correspondencia: Steffen Kevin Denis, LAINPA FCV-UNLP, 60 y 118 S/N, La Plata (CP: 1900), Buenos Aires, Argentina. E-mail: ksteffen@fcv.unlp.edu.ar

RESUMEN. El objetivo de este estudio fue determinar el comportamiento productivo en cabras Saanen mediante la caracterización de la curva de lactancia y el rendimiento de acuerdo al número de lactancia y al tipo de parto en un tambo semi-intensivo de la provincia de Buenos Aires. El hato caprino fue dividido en las diferentes categorías: cabras de primera lactancia $\left(1^{\circ} \mathrm{L}, \mathrm{n}=89\right)$; segunda lactancia $\left(2^{\circ} \mathrm{L}, \mathrm{n}=39\right)$, tercera lactancia $\left(3^{\circ} \mathrm{L}, \mathrm{n}=49\right)$; cuarta y quinta lactancia $\left(4^{\circ} \mathrm{L}\right.$ y $\left.5^{\circ} \mathrm{L}, \mathrm{n}=41\right)$ y última lactancia $(U \mathrm{~L}, \mathrm{n}=38)$. El tipo de parto fue clasificado en Simple (una cría, $n=120$ ) y Múltiple (dos o más crías, $n=136$ ). El control lechero se realizó evaluando el ordeño 2 veces por día cada $42 \pm 4$ días (método BT6) mediante ordeño mecánico con lactómetros Waikato. Mediante un análisis de varianza multifactorial, se evaluó la influencia del número de lactancia y tipo de parto sobre la lactancia completa (PLT). Se observó una diferencia estadísticamente significativa de los factores $(p=0,0074)$ y $(p=0,0179)$ respectivamente sobre la PLT. El análisis de media verificó diferencias significativas $(p<0,05)$ entre las cabras de $3^{\circ} \mathrm{L}$ y las de $1^{\circ} \mathrm{L}, 2^{\circ} \mathrm{L}$ y UL, así mismo, las de $4^{\circ} \mathrm{L}$ y $5^{\circ} \mathrm{L}$ respecto de $\mathrm{UL}(\mathrm{p}<$ $0,05)$. El tipo de parto observó diferencias significativas $(p<0,05)$ en la PLT entre las cabras múltiples y simples. El comportamiento productivo de cabras Saanen de un establecimiento caprino semi-intensivo de la provincia de Buenos Aires, asociado al número de lactancia y el tipo de parto registraron diferencias en la producción láctea.

SUMMARY. Characterization of the lactation and yield curve in Saanen goats from a dairy farm in the province of Buenos Aires, Argentina. The objective of this study was to determine the productive behavior in Saanen goats by characterizing the lactation curve and the yield according to the number of lactations and the type of delivery in a semi-intensive dairy farm in the province of Buenos Aires. The goat herd was divided into the different categories: first lactation goats (1st $L, n=89$ ); second lactation ( $2 n d L, n=39$ ), third lactation ( $3 r d L, n=49)$; fourth and fifth lactation $\left(4^{\circ} \mathrm{L}\right.$ and $5^{\circ} \mathrm{L}, \mathrm{n}=41$ ) and last lactation $(\mathrm{UL}, \mathrm{n}=38$ ). The type of delivery was classified as Simple (one kid, $n=120$ ) and Multiple (two or more kids, $n=136$ ). The milk control was carried out evaluating the milking twice a day every $42 \pm 4$ days (BT6 method) by means of mechanical milking with Waikato lactometers. Using a multifactorial analysis of variance, the influence of lactation number and type of delivery on full lactation (PLT) was evaluated. There was a statistically significant difference of the factors $(p=0.0074)$ and $(p=0.0179)$ respectively on the PLT. The mean analysis verified significant differences $(\mathrm{p}<0.05)$ between the goats of $3^{\circ} \mathrm{L}$ and those of $1^{\circ} \mathrm{L}, 2^{\circ} \mathrm{L}$ and $\mathrm{UL}$, likewise, those of $4^{\circ} \mathrm{L}$ and $5^{\circ} \mathrm{L}$ with respect to UL (p $<0.05)$. The type of delivery observed significant differences $(p<0.05)$ in the PLT between the multiple and simple goats. The productive behavior of Saanen goats from a semi-intensive goat farm in the province of Buenos Aires, associated with the number of lactations and the type of parturition, showed differences in milk production.

Palabras clave: producción láctea; Saanen; número de lactancia; tipo de parto; control lechero

Keywords: dairy production; Saanen; lactation number; type of parturition; milk control

\section{Introduction}

Históricamente la producción del ganado caprino en el mundo ha estado en manos de poblaciones con escasos recursos, que habitan áreas geográficas de bajo potencial productivo. En el mundo, según datos estadísticos se calcula que existen alrededor de 1045 millones de cabezas de caprinos domésticos (Capra aegagrus hircus), con un crecimiento poblacional entre el $14 \%$ y $18 \%$ de la cantidad de caprinos lecheros en los últimos 10 años (FAOSTAT, 2019). La leche caprina representa alrededor del 2,2\% de la producción mundial de leches, 
aumentando $15 \%$ en los últimos 10 años con un total aproximado de 19 millones de toneladas (FAOSTAT, 2019).

Se calcula que en Argentina existen 4.860 .000 cabezas, las que en su mayoría se encuentran distribuidas en hatos con menos de 500 animales y en alrededor de 47.404 establecimientos. Neuquén, Mendoza, Chaco y Santiago del Estero son las provincias que concentran la mayor cantidad de cabezas y establecimientos, con alrededor del $59 \%$ de la existencia caprina nacional (SIGSA, 2017). En los últimos años se han instalado en varias regiones, pequeños y medianos emprendimientos lácteos caprinos que han logrado incrementar la eficiencia productiva de los sistemas mediante la incorporación de mejoramiento genético, insumos, equipamiento y tecnologías asociados a prácticas productivas intensivas (AACREA, 2005; Paz, 2006; Ghibaudi et al., 2018). En Argentina, la leche de cabra es un producto regional apreciado desde el punto de vista nutricional y también funcional porque constituye un baluarte cultural y artesanal, tanto como actividad económica de sustento de productores como para autoconsumo (Martínez y Suárez, 2018). En nuestro país, existen alrededor de 200 tambos, con una población de aproximadamente 10.000 caprinos lecheros (SIGSA, 2017). En la provincia de Buenos Aires, en los últimos años se viene desarrollando la producción láctea caprina, para la fabricación de quesos artesanales y quesos gourmet, en cercanía de los grandes centros urbanos, facilitando la comercialización regional de productos con alto valor agregado e identidad propia (AACREA, 2005; Paz, 2006; Ghibaudi et al., 2018)

La raza lechera Saanen y sus cruzas son las predominantes en el país, seguidas por la Anglo Nubian y otras como Toggenburg, Alpina Francesa y Británica en menor escala (Paz, 2006; Martínez y Suárez, 2018; FAOSTAT, 2019). En Argentina, existen estudios sobre la producción media diaria de leche para la raza Saanen, oscilando entre $0,85-1,85 \mathrm{~kg} / \mathrm{cabra} / \mathrm{día}$, infiriendo promedios inferiores a los $400 \mathrm{~kg}$ por lactancias de entre 210 - 270 días, según método implementado para determinar la curva de lactancia (Paz et al., 2007; Frau et al., 2010; Frau et al., 2013; Martínez et al., 2018; Martínez y Suárez, 2018; Suárez y Martínez, 2019). En otros países, para la raza Saanen se registraron producciones superiores a $500 \mathrm{~kg} /$ lactancia como así también respecto a los rindes diarios de leche $(2,3$ a 3,1 L/d), (Gipson y Grossman, 1989; Soares Filho et al., 2001; Olivier et al., 2005; Mioč et al., 2008; Torres-Vázquez et al., 2010; Irano et al., 2012; Lôbo et al., 2017).

Se han propuesto diversos modelos matemáticos específicos para ajustar la producción lechera caprina (Gipson y Grossman, 1989; Gipson y Grossman, 1990; Fernández et al., 2002; Macciotta et al., 2005; Takma et al., 2009). Uno de los más utilizados es el método de Fleischmann o del día centrado (Takma et al., 2009; ICAR, 2018). La curva de lactancia es la representación matemática de la respuesta fisiológica de la producción de leche durante el período que dura el ordeño (Martínez et al., 2018). Su conocimiento, permite predecir el desempeño futuro de los animales, la producción total de leche, el tiempo en alcanzar el pico y la persistencia de la producción y efectuar ajustes de hembras con lactancias incompletas (Gipson y Grossman, 1989; Fernández et al., 2002; Arnal, 2018). Ésta es una importante herramienta en programas de mejoramiento genético, permitiendo la selección de hembras en base a producción de leche, optimizar el manejo nutricional y reproductivo de los animales en lactancia (Gipson y Grossman, 1989; Min et al., 2005; Goetsch et al., 2011; Arnal, 2018). Dado que el manejo nutricional conlleva gran parte de los costos de producción, es importante conocer las diferentes etapas de la curva de lactancia (primero, segundo y tercer tercio) en la que se encuentran los animales para, en función de ésta, hacer un uso más eficiente de la alimentación y adoptar diferentes estrategias de manejo (Min et al., 2005; Goetsch et al., 2011). Además, puede ayudar a identificar animales enfermos antes de que aparezcan signos clínicos, como aquellos animales con producción disminuida de leche por mastitis subclínica (Gipson y Grossman, 1989; Takma et al., 2009). Varios autores concluyeron que la forma de la curva de lactancia se ve afectada por diversos factores, tales como: componente racial o genotipo, la dieta, las condiciones climáticas, el período de lactancia, el año y la época de parto, número y el tipo de parto (Gipson y Grossman, 1989; Gipson y Grossman, 1990; Paz et al., 2007; Salvador y Martínez, 2007; Mioč et al., 2008; Frau et al., 2010; Torres-Vázquez et al., 2010; Irano et al., 2012; Bolacali y Küçük, 2012; Rojo-Rubio et al., 2016; Lôbo et al., 2017; Zamuner et al., 2020).

El objetivo de este estudio fue determinar el comportamiento productivo de cabras Saanen mediante la caracterización de la curva de lactancia y el rendimiento de acuerdo al número de lactancia y al tipo de parto en un tambo semi-intensivo de la provincia de Buenos Aires.

\section{Materiales y métodos}

El presente trabajo se llevó a cabo en un tambo caprino comercial, con elaboración propia de diferentes tipos de quesos, ubicado en el partido de General Paz $\left(35^{\circ} 16^{\prime} 00^{\prime \prime} \mathrm{S} 58^{\circ} 24^{\prime} 00^{\prime \prime} \mathrm{O}\right)$ en la provincia de Buenos Aires, Argentina. Se utilizaron registros de 4 a 6 controles lecheros de una lactancia (2019-2020) correspondiente a 256 cabras (Capra aegagrus hircus) de raza Saanen. El hato caprino fue dividido en las diferentes categorías: cabras de primera lactancia $\left(1^{\circ} \mathrm{L}, \mathrm{n}=89\right)$; cabras de segunda lactancia $\left(2^{\circ} \mathrm{L}, \mathrm{n}=39\right)$, de tercera lactancia $\left(3^{\circ} \mathrm{L}, \mathrm{n}=49\right)$; de cuarta y quinta lactancia $\left(4^{\circ} \mathrm{L}\right.$ y $5^{\circ} \mathrm{L}, \mathrm{n}=41$ ) y de última lactancia (UL, $n=38$ ), con fecha de parto entre Julio-Agosto de 2019. En relación al tipo de parto se clasificó en Simple (una cría, $n=120$ ) y Múltiple (dos o más crías, $n=136$ ). El sistema de manejo 
fue semi-intensivo y la alimentación a lo largo del período de evaluación para todo el hato fue de base pastoril con suplementación: $1 \mathrm{~kg} /$ animal/día de silo de maíz, $1 \mathrm{~kg} /$ animal/día alimento balanceado (16\% proteína bruta) y $6 \mathrm{~h}$ de pastoreo sobre una pastura implantada consociada con base de trébol blanco. El manejo del pastoreo fue mediante el uso de franjas diarias asignadas en función de la disponibilidad del forraje. Las cabras disponían de agua ad libitum y bloques estándar de minerales y oligoelementos. En relación al manejo reproductivo, los animales recibieron servicio durante la estación de verano otoño (Febrero-Marzo), concentrándose los partos aproximadamente en 30 días durante la época invernal (Julio-Agosto). A finales del 2017 el establecimiento incorporó un macho puro de pedigree de raza Saanen importado de Nueva Zelanda. El tambo realiza los controles periódicos obligatorios de brucelosis y tuberculosis sujetos a los protocolos correspondientes. Cada dos meses se realizó el diagnóstico coproparasitológico cuantitativo (HPG) mediante la técnica de McMáster modificada y el tratamiento de los animales que fuera necesario con monepantel: 3,75 $\mathrm{mg} / \mathrm{kg}$, vía oral.

El control lechero se realizó controlando el ordeño 2 veces por día cada $42 \pm 4$ días por personal técnico del establecimiento con supervisión veterinaria no oficial (método BT6) (ICAR, 2018), mediante ordeño mecánico. Los controles se realizaron de manera matutina a las 7 am y vespertina a las $16 \mathrm{pm}$. El primer control se realizó a partir de los $35 \pm 5$ días postparto. Los cabritos fueron destetados a las 48-72 h después de la ingesta de calostro y criados en lactancia artificial. Según ICAR 2018, se estableció la duración convencional de lactancia entre 210 y 240 días para considerarla lactancia completa. Las primíparas cuantificaron de 4 a 5 y las multíparas de 5 a 6 controles lecheros. Para la evaluación de la producción láctea individual (L), se utilizaron lactómetros MKV para cabras (Waikato, Nueva Zelanda). Los datos obtenidos mediante los controles lecheros fueron convertidos a kilogramos $(\mathrm{kg})$ estimando la densidad (d) de la leche para la raza Saanen (Densidad= 1,033 g/ml) (Frau et al.,2010; ICAR, 2018).

Para estimar la curva de lactancia se utilizó el método de Fleischmann aprobado por el Internationa Committee for Animal Recording - section 16 . Guidelines for performance recording in dairy sheep and dairy goats (ICAR, 2018). Fórmulas para producción de leche según el método de Fleischmann:

$$
\begin{aligned}
& \text { Producción }=\sum_{\substack{\mathrm{n} \text { - controles } \\
\text { Donde: }}}^{(\mathrm{pi}+\mathrm{pi}+1)} \times(\mathrm{di}+1-\mathrm{di}) \\
& 2
\end{aligned}
$$

-pi, es la producción ( $\mathrm{kg}$ de leche estandarizada) en el control número $\mathrm{i}$, donde $\mathrm{i}$ va de $0 \mathrm{a}$.

-di, son los días que la cabra lleva en lactación cuando se realiza el control número i
-La estandarización de la producción láctea total (PLT) de las cabras se realizó a 210 - 240 días.

Se realizó un análisis de varianza, donde se consideraron como factores, el número de lactancia y el tipo de parto, y se relacionó con la producción láctea (ANOVA multifactorial - Stat graphic Centurion XVII). Las diferencias se consideraron significativas para valores de $p \leq 0,05$ y tendencia de asociación para valores de $p$ entre 0,05 y 0,10 .

\section{Resultados y discusión}

En la Figura 1 se observa la lactancia completa (PLT) de cabras primíparas y multíparas, según el método de Fleischmann. Se observó un aumentó hasta el día $76 \pm 4$ días postparto de la producción láctea, lo que concuerda con el pico de lactancia para luego disminuir en los controles sucesivos.

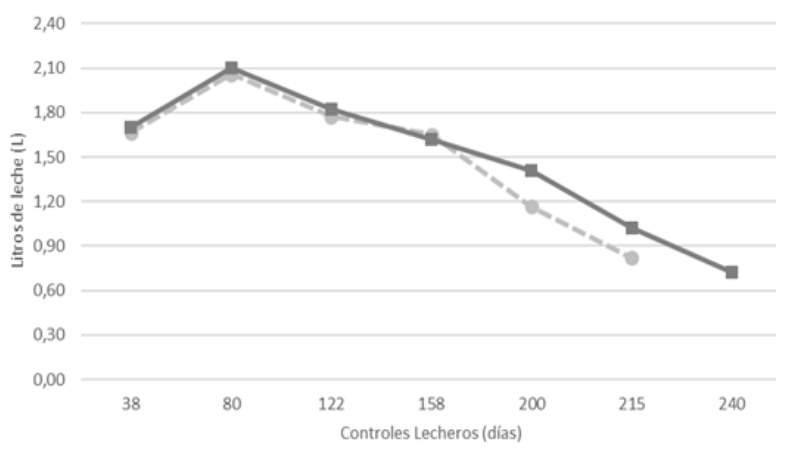

- 4 -Cabras primíparas (n:89) $\quad$-Cabras multiparas (n:167)

Figura 1. Producción de litros de leche de cabras de raza Saanen ajustada a lactancia completa (PLT) según el método de Fleischmann.

Se realizó el análisis de ANOVA multifactorial, según el número de lactancia y el tipo de parto, observándose una diferencia estadísticamente significativa sobre la PLT de cada uno de los factores $(p=0,0074)$ y $(p=$ 0,0179 ) respectivamente (Tabla 1 ).

Mediante el análisis de media se observaron diferencias significativas $(p<0,05)$ entre las cabras de tercera lactancia $553,51 \pm 18,552 \mathrm{~L}(571,77 \pm 19,164 \mathrm{~kg})$ y las de primera $476,08 \pm 26,919 \mathrm{~L}(491,79 \pm 27,807 \mathrm{~kg})$, segunda $474,10 \pm 26,113 \mathrm{~L}(489,74 \pm 26,974 \mathrm{~kg})$ y última lactancia 429,73 $\pm 24,180 \mathrm{~L}(443,91 \pm 24,977 \mathrm{~kg})$, así mismo, las cabras de cuarta y quinta lactancia $504,47 \pm$ $26,308 \mathrm{~L}(521,12 \pm 27,176 \mathrm{~kg})$ respecto de las cabras de última lactancia $(p<0,05)$ (Tabla 2, Figura 2).

En relación al tipo de parto se verificó una diferencia significativa $(p<0,05)$ en la producción láctea total (PLT) entre las cabras con parto múltiple $512,03 \pm 14,284 \mathrm{~L}$ $(528,92 \pm 14,755 \mathrm{~kg})$ y simple $463,13 \pm 17,695 \mathrm{~L}(478,41$ $\pm 18,278 \mathrm{~kg}$ ) (Tabla 2, Figura 3). 
Tabla 1. Análisis de Varianza multifactorial para Lactancia Completa (PLT). GL: Grados de libertad; A: Grupo N Lactancia; B: Grupo N Cría; AB: N Lactancia y NCría. Razón-F: Razón de Fisher. Valor-P: Valores $(<0,05)$ indican diferencia significativa.

\begin{tabular}{|c|c|c|c|c|c|}
\hline Ítem & $\begin{array}{l}\text { Suma } \\
\text { cuadrados }\end{array}$ & GL & $\begin{array}{l}\text { Cuadrado } \\
\text { medio }\end{array}$ & $\begin{array}{l}\text { Razón- } \\
\text { F }\end{array}$ & $\begin{array}{l}\text { Valor- } \\
\text { P }\end{array}$ \\
\hline $\begin{array}{l}\text { EFECTOS } \\
\text { PRINCIPALES }\end{array}$ & & & & & \\
\hline $\begin{array}{l}\text { A: Grupo } \mathbf{N}^{\circ} \\
\text { Lactancia }\end{array}$ & 381703 & 4 & 95425,7 & 3,58 & 0,0074 \\
\hline B: Grupo $N^{\circ}$ Cría & 151519 & 1 & 151519 & 5,68 & 0,0179 \\
\hline \multicolumn{6}{|l|}{ INTERACCIONES } \\
\hline$A B$ & 89247,7 & 4 & 22311,9 & 0,84 & 0,5029 \\
\hline RESIDUOS & $6,55867 \mathrm{E} 6$ & 246 & 26661,3 & & \\
\hline $\begin{array}{l}\text { TOTAL } \\
\text { (CORREGIDO) }\end{array}$ & $7,2592 \mathrm{E} 6$ & 255 & & & \\
\hline
\end{tabular}

En la cuenca lechera de Santiago del Estero, la producción media diaria de leche para la raza Saanen, fue de $1,27 \pm 0,27 \mathrm{~kg} / \mathrm{d}$ (Frau et al., 2010); 1,44 kg/d (Frau et al., 2013) y 0,94 $\pm 0,05 \mathrm{~kg} / \mathrm{d}$ para las razas mestiza Saanen (Paz et al., 2007). Para esta última raza las producciones por lactancia fueron de 197,54 $\pm 11,36 \mathrm{~kg}$ para los 210 días de lactancia. Recientemente, Suárez y Martínez, (2019) estudiaron diversos tambos caprinos del noroeste argentino con diferentes razas, para este caso, se tomó como referencia los establecimientos con raza Saanen y mestiza de Saanen de dos tambos de Salta y dos de Santiago del Estero. Se observó que la producción de leche fue $1,28 \mathrm{~L} / \mathrm{d} ; 1,80 \mathrm{~L} / \mathrm{d}$ y $1,05 \mathrm{~L} / \mathrm{d}$; $0,99 \mathrm{~L} / \mathrm{d}$, respectivamente y con una duración promedio de la lactancia por cabra de $221 \pm 37$ días. En el presente estudio, en la provincia de Buenos Aires, la producción fue superior a lo reportado por estos autores, la media diaria osciló entre 2,08 $\pm 0,097 \mathrm{~L} / \mathrm{d}$ $(2,14 \pm 0,100 \mathrm{~kg} / \mathrm{d})$ y los promedios por lactancias ajustados a $210-240$ días fueron de 487,583 $\pm 24,413 \mathrm{~L}$ $(503,67 \pm 25,218 \mathrm{~kg})$. Esta diferencia en la producción láctea de la raza Saanen entre diferentes provincias del país, podría deberse a diferencias en el desarrollo del sistema de producción, cruzamientos y limitantes alimenticias en el noroeste Argentino. Por lo tanto, el potencial productivo de las cabras Saanen estaría condicionado por diversos factores como, el cruzamiento con biotipos criollos y la falta de selección genética, condiciones climáticas, poca tecnificación con escasa inversión de infraestructura, deficiente alimentación basada sobre la curva forrajera natural, los efectos de las enfermedades y al tipo de mano de obra utilizada.
Tabla 2. Referencias: $1^{\circ} \mathrm{L}$ : Cabras de primera lactancia; $2^{\circ} \mathrm{L}$ : Cabras de segunda lactancia; $3^{\circ} \mathrm{L}$ : Cabras de tercera lactancia; $4^{\circ} \mathrm{L}$ y $5^{\circ} \mathrm{L}$ : Cabras de cuarta y quinta lactancia; UL: Cabras de última lactancia; EE: Error Estándar. Letras diferentes indican diferencias significativas.

\begin{tabular}{|c|c|c|c|}
\hline \multicolumn{4}{|l|}{ Ítem } \\
\hline Grupo $N^{\circ}$ Lactancia & Casos (n) & $\begin{array}{l}\text { Media (L) } \\
\text { Media (kg) }\end{array}$ & $\mathrm{EE}$ \\
\hline $1^{\circ} \mathrm{L}$ & 89 & $\begin{array}{l}476,08^{a b} \\
491,79^{a b}\end{array}$ & $\begin{array}{l}26,919 \\
27,807\end{array}$ \\
\hline $2^{\circ} \mathrm{L}$ & 39 & $\begin{array}{l}474,10^{a b} \\
489,74^{a b}\end{array}$ & $\begin{array}{l}26,113 \\
26,974\end{array}$ \\
\hline $3^{\circ} \mathrm{L}$ & 49 & $\begin{array}{l}553,51^{c} \\
571,77^{c}\end{array}$ & $\begin{array}{l}18,552 \\
19,164\end{array}$ \\
\hline $4^{\circ} \mathrm{L}$ y $5^{\circ} \mathrm{L}$ & 41 & $\begin{array}{l}504,47^{b c} \\
521,12^{\text {bc }}\end{array}$ & $\begin{array}{l}26,308 \\
27,176\end{array}$ \\
\hline UL & 38 & $\begin{array}{l}429,73^{a} \\
443,91^{a}\end{array}$ & $\begin{array}{l}24,180 \\
24,977\end{array}$ \\
\hline Grupo N Cría & & & \\
\hline Múltiple & 136 & $\begin{array}{l}512,03^{a} \\
528,92^{a}\end{array}$ & $\begin{array}{l}14,284 \\
14,755\end{array}$ \\
\hline Simple & 120 & $\begin{array}{l}463,13^{b} \\
478,41^{b}\end{array}$ & $\begin{array}{l}17,695 \\
18,278\end{array}$ \\
\hline Total & 256 & $\begin{array}{l}487,58 \\
503,67\end{array}$ & $\begin{array}{l}24,413 \\
25,218\end{array}$ \\
\hline
\end{tabular}

Lôbo et al. (2017) trabajando con cabras en el sureste de Brasil, demostraron que los promedios de producción total de leche (ajustado a 305 días) y producción media diaria para la raza Saanen fueron de $940,38 \pm 30,06 \mathrm{~kg} ; 3,13 \pm 0,05 \mathrm{~kg} / \mathrm{d}$ respectivamente. Olivier et al. (2005) en Sudáfrica; Torres-Vázquez et al. (2010) en México y Mioč et al. (2008) en Croacia, obtuvieron cifras promedio de producción total de leche, para la misma raza, de $841,1 \mathrm{~kg}$ (ajustado a 246 días de lactancia), $933 \mathrm{~kg}$ (ajustado a 305 días de lactancia) y $720,08 \pm 12,49 \mathrm{~kg}$ respectivamente. Estos promedios están muy por encima de nuestros resultados para la raza Saanen en Buenos Aires, como lo demuestra la Tabla 2. Los mismos se asemejan a los encontrados por Irano et al. (2012) para las razas Alpina y Saanen del sudeste de Brasil con 511,7 kg y 2,4 kg de producción media diaria (ajustado a 211 días de lactancia) y por Zamuner et al. (2020) en Australia, que fue $519 \pm 7,3 \mathrm{~kg}$ para la producción total de leche ajustada 270 días de lactancia. En nuestro trabajo se 
demostraron valores mayores de producción láctea a los reportados por Bolacali y Küçük (2012) y Lôbo et al. (2017), con $383,05 \pm 6,74 \mathrm{~kg}$ por lactancia ajustado a 270 días y 1,37 $\pm 0,02 \mathrm{~kg} / \mathrm{d}$ en Turquía y $362,05 \pm 21,28$ $\mathrm{kg}$ ajustado a 305 días, y 1,56 $\pm 0,03 \mathrm{~kg} / \mathrm{d}$ en el noreste de Brasil, respectivamente.

En relación al número de lactancia, la producción de leche aumenta hasta la tercera y cuarta para luego disminuir en las sucesivas lactancias. Este resultado se corrobora con la mayoría de los trabajos encontrados en la literatura sobre cabras lecheras (Soares Filho et al., 2001; Olivier et al., 2005; Mioč et al., 2008; TorresVázquez et al., 2010; Bolacali y Küçük, 2012; Irano et al., 2012; Lôbo et al., 2017; Zamuner et al., 2020), en coincidencia con lo observado en nuestro trabajo. En disidencia con Garcés et al. (2004), trabajando con cabras Saanen, observaron que las cabras multíparas obtuvieron igual producción lechera que las primíparas.

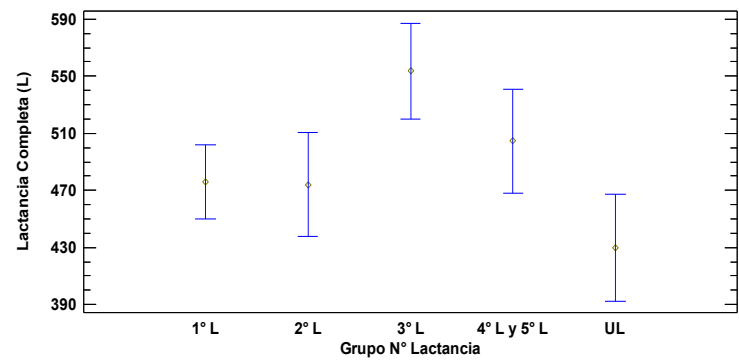

Figura 2. Media para Lactancia completa (PLT) según el número de lactancia. Las barras de error representan intervalos de confianza del $95 \%$. Referencias: $1^{\circ} \mathrm{L}$ : Cabras de primera lactancia; $2^{\circ} \mathrm{L}$ : Cabras de segunda lactancia; $3^{\circ} \mathrm{L}$ : Cabras de tercera lactancia; $4^{\circ} \mathrm{L}$ y $5^{\circ} \mathrm{L}$ : Cabras de cuarta y quinta lactancia; UL: Cabras de última lactancia.

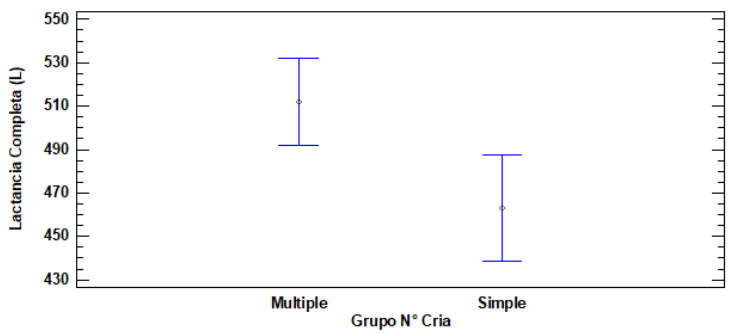

Figura 3. Media para Lactancia completa (PLT) según el tipo de parto (múltiple o simple). Las barras de error representan intervalos de confianza del 95\%. Referencias: Múltiple: Cabras con dos o más crías; Simple: Cabras con una cría.

Respecto al tipo de parto, las cabras con partos múltiples $512,03 \pm 14,284 \mathrm{~L}(528,92 \pm 14,755 \mathrm{~kg})$ produjeron $10 \%$ más de leche que las cabras de partos simples $463,13 \pm 17,695 \mathrm{~L}(478,41 \pm 18,278 \mathrm{~kg})$, y en concordancia con Paz et al. (2007), quienes observaron que las cabras de parto simple produjeron significativamente menos que las de parto múltiple $(149,60$ $\pm 11,25 \mathrm{~kg}$ y $191,68 \pm 9,93 \mathrm{~kg}$, respectivamente). De un modo similar, pero sin diferencias significativas, Soares Filho et al. (2001) identificaron que cabras con partos dobles y triples observaron producciones medias de leches mayores que aquellas de partos simples $(519,10$ $\mathrm{kg}, 560,44 \mathrm{~kg}$ y $498,89 \mathrm{~kg}$, respectivamente), En el mismo sentido, Bolacali y Küçük (2012), Rojo-Rubio et al. (2016), Lôbo et al. (2017), Zamuner et al. (2020), identificaron una productividad significativamente mayor en los partos múltiples, probablemente la presencia de una directa y estrecha correlación entre la producción de leche y la masa placentaria expresada en gramos, atribuyendo dicho efecto a una correlación positiva entre la cantidad de lactógenos placentarios y el número de fetos (Haydn et al., 1979).

La forma de la curva de lactancia se ve afectada por diversos factores (Gipson y Grossman, 1989; Gipson y Grossman, 1990; Soares Filho et al., 2001; Paz et al., 2007; Salvador y Martínez, 2007; Mioč et al., 2008; Frau et al., 2010; Goetsch et al., 2011; Bolacali y Küçük, 2012; Irano et al., 2012; Rojo-Rubio et al., 2016; Lôbo et al., 2017; Arnal, 2018; Zamuner et al., 2020), como quedó demostrado en el establecimiento según las variables analizadas.

En función a los resultados obtenidos en el presente trabajo, se puede concluir que el comportamiento productivo de cabras Saanen de un establecimiento caprino de la provincia de Buenos Aires en condiciones semi-intensivas, asociado con el número de lactancia y el tipo de parto registraron diferencias en la producción láctea.

\section{Agradecimientos}

Agradecemos al Tambo caprino "La Capriola" y su encargado Samuel por la colaboración y su predisposición. El estudio de estos animales fue aprobado por el Comité Institucional de Cuidado y Uso de Animales de Laboratorio (CICUAL), FCV-UNLP (protocolos 97-1-19T) y avalado mediante el consentimiento informado del propietario para su publicación.

\section{Referencias}

Asociación Argentina de Consorcios Regionales de Experimentación Agrícola (AACREA) 2005. Agroalimentos Argentinos II. 247 pp.

Arnal M, Robert-Granié C, Larroque H. 2018. Diversity of dairy goat lactation curves in France. J. Dairy Sci. 101: 11040-11051.

Bolacali M, \& Küçük M. 2012. Fertility and milk production characteristics of Saanen goats raised in Muș Region. Kafkas Üniversitesi Veteriner Fakültesi Dergisi 18: 351-358.

FAOSTAT. 2019. Food and Agriculture Organization of the United Nations. Statistics data base. [Online] http://www.fao.org/faostat/en/\#data/QA.

Fernández C, Sanchez A, Garces C. 2002. Modeling the lactation curve for test-day milk yield in Murciano-Granadina goats. Small Rumin. Res. 46: 29-41. 
Frau S, Togo J, Pece N, Paz R, Font G. 2010. Estudio comparativo de la producción y composición de leche de cabra de dos razas diferentes en la provincia de Santiago del Estero. Revista de la Facultad de Agronomía, La Plata 109: 9-15.

Frau S, Font G, Paz R, Pece N. 2013. Composición fisicoquímica y calidad microbiológica de leche de cabra producida en la provincia de Santiago del Estero (Argentina). Asociación Latinoamericana de Producción Animal. Archivos Latinoamericanos de Producción Animal 21: 1-13.

Garcés R, Boza L, Acevedo P, Brandl E, Bruckmaier R, Luis López J. 2004. Persistence index and description of first 100 days of the lactation curve of primiparous and multiparous Saanen goats maintained in confinement. Agricultura Técnica (Chile) 64: 319-326.

Ghibaudi M, Simonetti L, Ponce V, De Lima A, Feoli E, Flor S, López C. 2018. Introducción a la lechería caprina. Revista de Divulgación Técnica Agropecuaria, Agroindustrial y Ambiental. Facultad de Ciencias Agrarias - UNLZ 5: 50-60.

Gipson TA, Grossman M. 1989. Dyphasic analysis of lactation curves in dairy goats. J. Dairy Sci. 72: 1035-1044.

Gipson TA, Grossman M. 1990. Lactation curves in dairy goats: a review. Small Rumin. Res. 3: 383-396.

Goetsch, A. L., Zeng, S. S., \& Gipson, T. A. 2011. Factors affecting goat milk production and quality. Small Rumin. Res. 101: 55-63.

Hayden TJ, Thomas CR, Forsyth IA. 1979. Effect of Number of Young Born (Litter Size) on Milk Yield of Goats: Role for Placental Lactogen. J. Dairy Sci. 62: 53-57.

ICAR. 2018. The International Committee for Animal Recording. International agreement of recording practices. Approved by the General Assembly held in Berlin, Germany, on May 2014. Section 2.3 - ICAR rules, standards and guidelines for milk recording in goats, pp. 77-87.

Irano N, Bignardi AB, Rey FSB, Teixeira IAMA, Albuquerque LG. 2012. Parâmetros genéticos para a produção de leite em caprinos das raças Saanen e Alpina. Revista Ciência Agronômica 43: 376-381.

Lôbo AMBO, Lôbo RNB, Facó O, Souza V, Alves AAC, Costa AC, Albuquerque MAM. 2017. Characterization of milk production and composition of four exotic goat breeds in Brazil. Small Rumin. Res. 153: 9-16.

Macciotta NPP, Fresi P, Usai G, Cappio-Borlino A. 2005. Lactation curves of Sarda breed goats estimated with test-day models. J. Dairy Res. 72: 470-475.

Martínez GM, Suárez VH. 2018. Biotipos con aptitud lechera presentes en Argentina. En: Lechería Caprina: producción, manejo, sanidad, calidad de leche. 1a. edición. Ediciones INTA, pp. 29-33.

Martínez G, Léon Jurado J, Suárez V, Barba Capote C. 2018. Determinación de la curva de lactancia de cabras Saanen del noroeste argentino. FAVE Sección Ciencias Veterinarias 17: 611.
Min BR, Hart SP, Sahlu T, Satter LD. 2005. The effect of diets on milk production and composition, and on lactation curves in pastured dairy goats. J. Dairy Sci. 88: 2604-2615.

Mioč M, Zvonimir P, Ivan V, Zdravko B, Dubravka S. 2008. Factors affecting goat milk yield and composition. Mljekarstvo / Dairy 58: 305-313.

Olivier JJ, Cloete SWP, Schoeman SJ, Muller CJC. 2005. Performance testing and recording in meat and dairy goats, Small Rumin. Res. 60: 83-93.

Paz R. 2006. El Complejo Agroindustrial Lechero Caprino Argentino. Iniciativas para su desarrollo y mejora de la competitividad global. Revista Interdisciplinaria de Estudios Agrarios $\mathrm{N}^{\circ} 24$.

Paz R, Togo JA, López C. 2007. Evaluación de parámetros de producción de leche en caprinos Santiago del Estero, (Argentina) Revista Científica, FCV-LUZ 17: 161-165.

Rojo-Rubio R, Kholif AE, Salem AZM, Mendoza GD, Elghandour, MMMY, Vazquez-Armijo, JF, Lee-Rangel H. 2016. Lactation curves and body weight changes of Alpine, Saanen and AngloNubian goats as well as pre-weaning growth of their kids. J. Appl. Anim. Res. 44: 331-337.

Salvador A, Martínez G. 2007. Factores que Afectan la Producción y Composición de la Leche de Cabra: Revisión Bibliográfica Rev. Fac. Cs. Vet. UCV. 48: 61-76.

SIGSA. 2017. Dirección de Control de Gestión y Programas Especiales - Dirección Nacional de Sanidad Animal - SENASA. [Online] (https://www.argentina.gob.ar/senasa/caprinossector-primario)

Soares Filho G, McManus C, Mariante AS. 2001. Fatores genéticos e ambientais que influenciam algumas características de reprodução e produção de leite em cabras no Distrito Federal. Rev. Bras. Zootecnia 30: 133-140.

Suárez VH, Martínez GM. 2019. Características de los tambos caprinos comerciales y posibilidades de mejora genética en el noroeste argentino. Revista Veterinaria Argentina 36: 1-13.

Takma C, Akbaş Y, Taskin T. 2009. Modeling lactation curves of Turkish Saanen and Bornova goats. Asian J. Anim. Vet. Advances 4: 122-129.

Torres-Vázquez JA, Valencia-Posadas $M$, Castillo-Juárez $H$, Montaldo HH. 2010. Genetic and phenotypic trends for milk yield and milk composition traits of Saanen goats from Mexico, Rev. Mex. Cien. Pecuarias 1: 337-348.

Zamuner F, DiGiacomo K, Cameron AWN, Leury BJ. 2020. Effects of month of kidding, parity number, and litter size on milk yield of commercial dairy goats in Australia. J. Dairy Sci. 103: 954-964. 